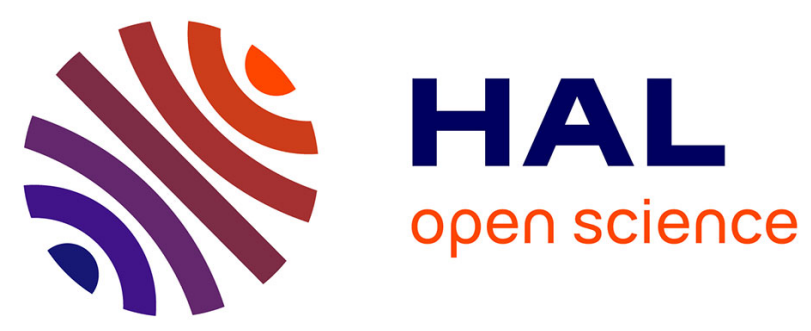

\title{
Le changement spatial dans l'industrie automobile en Europe centrale et orientale: entre effets d'héritage, cycles de vie et transition
}

Sébastien Bourdin, Anaïs Le Thiec, Bernard Elissalde

\section{To cite this version:}

Sébastien Bourdin, Anaïs Le Thiec, Bernard Elissalde. Le changement spatial dans l'industrie automobile en Europe centrale et orientale: entre effets d'héritage, cycles de vie et transition. Géocarrefour - Revue de géographie de Lyon, 2009, Numéro spécial "Quelle Europe vingt ans après la chute du Mur?" (Numéro Vol. 84/3), 13 p. 10.4000/geocarrefour.7438 . hal-00669085

\section{HAL Id: hal-00669085 https://hal.science/hal-00669085}

Submitted on 11 Feb 2012

HAL is a multi-disciplinary open access archive for the deposit and dissemination of scientific research documents, whether they are published or not. The documents may come from teaching and research institutions in France or abroad, or from public or private research centers.
L'archive ouverte pluridisciplinaire $\mathbf{H A L}$, est destinée au dépôt et à la diffusion de documents scientifiques de niveau recherche, publiés ou non, émanant des établissements d'enseignement et de recherche français ou étrangers, des laboratoires publics ou privés. 


\title{
Géocarrefour
}

Numéro Vol. 84/3 (2009)

Quelle Europe vingt ans après la chute du Mur?

Sébastien Bourdin, Anaïs Le Thiec et Bernard Elissalde

\section{Le changement spatial dans l'industrie automobile en Europe centrale et orientale : entre effets d'héritage, cycles de vie et transition}

\begin{abstract}
Avertissement
Le contenu de ce site relève de la législation française sur la propriété intellectuelle et est la propriété exclusive de l'éditeur.

Les œuvres figurant sur ce site peuvent être consultées et reproduites sur un support papier ou numérique sous réserve qu'elles soient strictement réservées à un usage soit personnel, soit scientifique ou pédagogique excluant toute exploitation commerciale. La reproduction devra obligatoirement mentionner l'éditeur, le nom de la revue, l'auteur et la référence du document.

Toute autre reproduction est interdite sauf accord préalable de l'éditeur, en dehors des cas prévus par la législation en vigueur en France.
\end{abstract}

\section{revues.org}

CENTRE POUR L'ÉDTION ÉECTRONIQUE OUVE

Revues.org est un portail de revues en sciences humaines et sociales développé par le CLEO, Centre pour l'édition électronique ouverte (CNRS, EHESS, UP, UAPV).

\section{Référence électronique}

Sébastien Bourdin, Anaïs Le Thiec et Bernard Elissalde, « Le changement spatial dans l'industrie automobile en Europe centrale et orientale : entre effets d'héritage, cycles de vie et transition », Géocarrefour [En ligne], Vol. 84/3 | 2009, mis en ligne le 04 janvier 2010. URL : http://geocarrefour.revues.org/index7438.html $\mathrm{DOI}$ : en cours d'attribution

Éditeur : Association des amis de la Revue de Géographie de Lyon http://geocarrefour.revues.org

http://www.revues.org

Document accessible en ligne à l'adresse suivante : http://geocarrefour.revues.org/index7438.html Ce document est le fac-similé de l'édition papier.

Cet article a été téléchargé sur le portail Cairn (http://www.cairn.info)

\section{CA I R N}

Chercher. Repérer. Avancer.

Distribution électronique Cairn pour Association des amis de la Revue de Géographie de Lyon et pour Revues.org (Centre pour l'édition électronique ouverte)

(c) Géocarrefour 


\section{Sébastien BOURDIN Anaïs LE THIEC Bernard ELISSALDE}

Université de Rouen UMR CNRS IDEES

\section{MOTS CLÉS}

Europe Centrale et Orientale, Roumanie, automobile, localisations, temporalités écologiques

\section{KEY WORDS}

Eastern and Central Europe, Romania, automobile sector, location, temporality

\section{Le changement spatial dans l'industrie automobile en Europe centrale et orientale : entre effets d'héritage, cycles de vie et transition}

\section{RÉSUMÉ}

La montée en puissance des nouveaux États membres de l'Union européenne dans la production automobile est replacée dans les processus à long terme de recherche de nouveaux espaces de consommation et de production par les entreprises de ce secteur. Analysé à travers les théories du cycle du produit et du modèle de Dünning, l'élargissement vers l'Europe centrale et orientale de I'industrie automobile pose la question de la nature de l'intégration en cours, associant des processus bien rôdés d'implantation industrielle aux héritages d'anciens territoires de production de l'ère communiste.

\section{ABSTRACT}

This paper aims to take into account the increasing weight of Eastern Europe in European car production. Through the product life-cycle theory and the Dunning model, recent foreign direct investment and the location of new plants are seen as new steps in enlarging the productive space of trans-national groups in the automobile sector.

Dans I'Union européenne, I'ouverture de nouveaux marchés à l'occasion de chaque nouvel élargissement s'accompagne d'une mobilité du capital sous forme d'investissements directs étrangers et/ou de restructurations d'anciens sites industriels. L'intérêt que suscite l'Europe centrale et orientale n'est plus aujourd'hui uniquement lié aux recompositions territoriales à l'œuvre actuellement dans ces pays, mais il a trait aussi aux interférences entre ces processus et la montée en puissance de la filière automobile, liées aux initiatives des grands groupes internationaux de ce secteur.

Le secteur automobile européen occupe une place prééminente à la fois sur la scène internationale (34 \% de la production automobile mondiale) et dans l'espace communautaire $3 \%$ du PIB communautaire et $7,5 \%$ de son industrie manufacturière). Cette industrie, symbole du régime d'accumulation fordiste et du mode de consommation de l'Europe occidentale, fait régulièrement I'objet d'études approfondies (Chanaron et Lung, 1995 ; Lung, 2002) notamment sous l'angle des stratégies des firmes dans le processus de globalisation des productions et des marchés. Elle retient ici notre attention comme exemple de tournant industriel à la fois pour les pays d'Europe centrale et orientale et pour l'équilibre interne de l'espace communautaire et du processus d'intégration des PECO (pays $d^{\prime} E$ urope centrale et orientale). Si représentative des dynamiques récentes de I'Union que puisse être cette évolution, ses caractéristiques tout comme le mode opératoire suivi par les acteurs concernés doivent être comparés à d'autres dynamiques industrielles, contemporaines de précédents élargissements de l'Union. Elles seront analysées à la lumière des cadres théoriques et de leurs implications géographiques, tels que ceux du cycle du produit de R.Vernon (Vernon, 1966) ou de I'ESP de J.H.Dunning (1988; 2001). Dans le séquençage localisation/délocalisation/relocalisation, assisterait-on, dans les PECO, à un nouvel élargissement de l'aire d'extension du fordisme et à l'apparition d'une sorte de néo-fordisme tardif dans des régions où les pénuries du système antérieur ne lui avait pas permis de s'épanouir?
Ou bien, est-on confronté à une hybridation entre ce régime et des héritages du mode de production socialiste ? L'article s'attachera plus particulièrement aux jeux des temporalités des héritages, des cycles de vie et de la transition économique pour interroger les recompositions spatiales du secteur automobile en Europe centrale et orientale et en Roumanie plus particulièrement.

\section{CYCLES ÉCONOMIOUES ET CONSTRUCTION DES ESPACES INDUSTRIELS EN EUROPE}

Organisation fordiste de la production et localisations industrielles

Les dynamiques spatiales accompagnant le développement du fordisme dans les économies développées durant les "Trente Glorieuses " ont donné lieu à des analyses (voir notamment Aydalot, 1976 ; Benko et Lipietz, 2000 ; Veltz, 1996) qui soulignaient que, si ce régime d'accumulation avait permis d'élargir des normes de consommation à l'ensemble de la société, il s'était accompagné de l'utilisation de différentiels de développement entre les régions d'un même pays, afin de mettre en place une division spatiale du travail fondée sur les niveaux de qualification et donc de salaires des personnels. L'ensemble de ce déploiement spatial fut baptisé " circuit de branche ". Durant les années 1990, certains auteurs cités plus haut et d'autres (Scott, 2001), influencés par l'école de la régulation, ont diagnostiqué la fin du régime d'accumulation fordiste dans les pays industrialisés et, par là même, la remise en cause des logiques de localisation des activités qui l'accompagnaient. Sans se situer complètement dans le cadre de cette approche, $M$. Porter et $d^{\prime}$ autres soulignèrent le rôle des économies d'agglomération, des externalités locales et détectèrent des logiques de clustering dans les nouvelles configurations des activités. Ces clusters d'entreprises appartenant à une même branche correspondent à des externalités locales dans le domaine des technologies, de la formation, de la coopération technique et des relations de sous-traitance. 
Les caractéristiques du déploiement spatial et la complexité des choix de localisation, balançant entre concentration et dispersion illustrent, dans l'automobile plus qu'ailleurs, les inflexions et les recompositions des stratégies des principaux groupes. Si, à la suite de ces auteurs, nous considérons que, sur le long terme, les industries produisent l'espace économique davantage qu'elles ne le subissent, cette affirmation nous conduira à nous interroger sur la nature des formes d'organisation de l'espace industriel créées par les investissements des firmes automobiles dans les PECO.

\section{Une nouvelle phase du cycle de vie de l'automobile européenne?}

Pour cerner la spécificité du cas européen, nous nous proposons de confronter cette multiplication des investissements du secteur automobile à partir des années 1990 dans les PECO à la théorie du cycle de vie du produit. Cette approche fut élaborée dans les années 1960 (Vernon, 1966) pour expliquer les changements dans les flux d'échanges internationaux et les délocalisations industrielles. Présenté comme un schéma phasé, le modèle débute par une phase de lancement d'un nouveau produit, incluant un fort contenu technologique, qui donne un avantage concurrentiel au pays innovant et qui s'appuie sur un marché domestique caractérisé par une société à hauts revenus. Lorsque le produit se standardise, au cours de la seconde phase (fabrication en grande série, réalisation d'économies d'échelle), que les coûts de production et les prix de vente diminuent, la sphère de consommation va s'élargir aux autres pays développés. La technologie se banalise jusqu'à la phase de maturité où le produit devient un bien de consommation courante pour toutes les catégories de population. Les firmes des pays développés tendent à transférer une partie de la production vers des pays moins développés où la main-d'œuvre est meilleur marché. Résultant de la localisation différenciée des activités et des marchés, les flux d'échange se renversent selon les phases du cycle. L'excédent commercial croît d'abord puis décroît, pour enfin laisser la place à un déficit commercial dans le pays " naisseur ".

La comparaison (fig. 1) des soldes des balances commerciales du secteur automobile de la France et de l'ensemble des PECO rend compte des translations des volumes de production. Traditionnellement excédentaire, la balance française des échanges automobiles s'infléchit à partir du début de la décennie 2000, non pas sous le coup d'un retournement de conjoncture, mais du fait de la part grandissante de la fabrication de véhicules des marques françaises hors de I'hexagone ${ }^{1}$. À l'inverse, le déficit initial des PECO dû à la diffusion de l'automobile dans de nouvelles couches de la population (importations de marques " occidentales", fig. 6) se réduit peu à peu au fur et à mesure de la montée en puissance des productions sur place des marques d'Europe de l'Ouest.

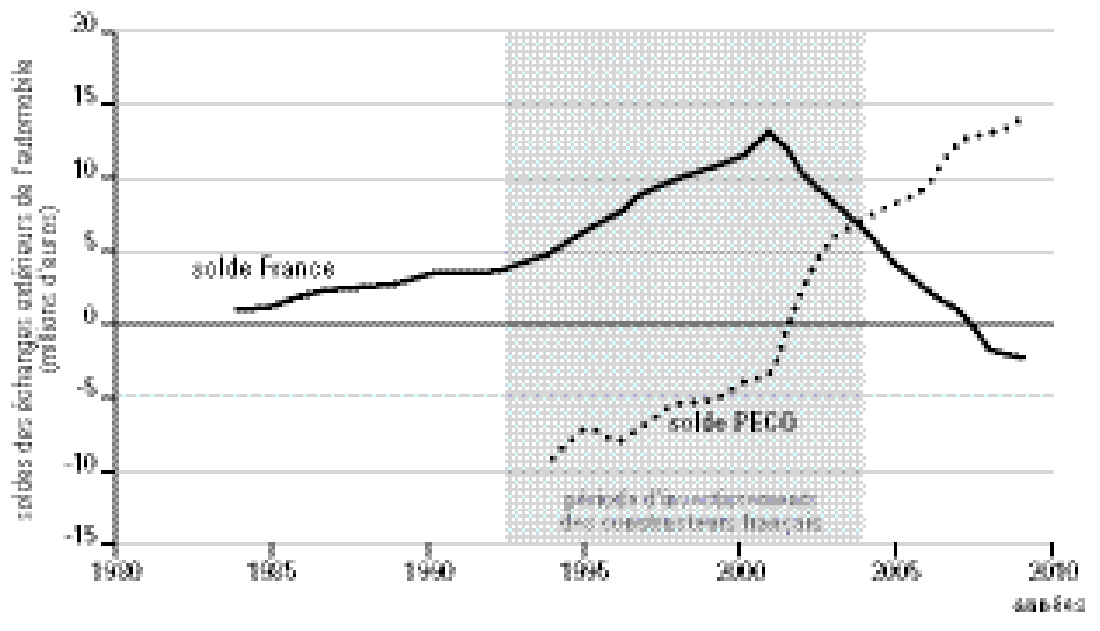

Cette théorie apporte en géographie un éclairage sur le processus spatio-temporel des spécialisations productives et des consommations de biens, ainsi que sur les implications dans la structure des échanges entre catégories de pays ou de région, pendant le déroulement du cycle. Derrière ces spécialisations qui, en apparence, ne sont séparées que par des intervalles de temps, émerge une hiérarchie spatiale, induite par la hiérarchie des technologies. Néanmoins, plus que l'image d'un cycle unique, les trajectoires des localisations des unités de production du secteur automobile vont révéler, sur la longue période, un séquençage en plusieurs cycles partiels, plusieurs fois recommencés, sans que les phases du cycle précédent soient totalement achevées.

\section{Cycle du produit, espace intégré et concurrence territoriale au sein de l'UE}

Dans un espace économiquement et commercialement intégré comme l'est devenue I'Union européenne, se sont développés des mécanismes de concurrence interne entre les régions européennes dont ont su tirer parti les nouveaux États membres. L'intégration fut d'abord réalisée dans le domaine des échanges commerciaux et conduisit à la formation d'un ensemble territorial où les différentes composantes fonctionnèrent de manière interdépendante. Cette situation d'intensification des relations, des échanges et des flux s'étendit géographiquement, au fil des élargissements, à de nouvelles régions dont certaines présentent des $\mathrm{PIB} /$ habitant inférieurs de moitié à la moyenne communautaire.

Spécialisée et organisée initialement en fonction de systèmes monétaires et fiscaux nationaux, chaque région est désormais confrontée à de nouveaux espaces utilisant la même monnaie ou des monnaies nationales indexées sur l'euro. L'économiste R. Mundell a théorisé, en 1971, l'idée de zones monétaires optimales, permettant de comprendre comment, en cas de ralentissement de la conjoncture pour l'un et de croissance pour I'autre, deux pays faisant partie d'un même ensemble monétaire devront utiliser $d^{\prime}$ autres variables que la dévaluation, telle la flexibilité du marché du travail (chômage, rigidité des salaires, mobilité du travail) comme substitut au mécanisme des taux de change. Le pays qui possède un avantage concurrentiel sur l'autre
Figure 1 : cycle du produit et échanges dans le secteur automobile entre la France et les PECO.

1 - La détérioration de la balance commerciale automobile n'est pas due non plus à un recul des positions commerciales puisque les parts de marché des constructeurs nationaux sont restées identiques. 
2 - À la veille de 1989, le nombre de voiture pour 1000 habitants n'était que de 117 pour les pays de l'Est contre 360 dans I'Union européenne.

3 - Conseil d'assistance économique mutuelle ou Comecon. (salaires, fiscalité) va voir sa demande et sa balance courante ne cesser d'augmenter, tandis que l'autre va voir ses industries se délocaliser et son chômage gonfler, sauf s'il se spécialise dans des secteurs à forte valeur ajoutée et réalise d'importants gains de productivité grâce à l'investissement.

\section{LE (RE)DÉPLOIEMENT DE L'INDUSTRIE AUTOMOBILE EN EUROPE CENTRALE ET ORIENTALE}

Depuis son origine en Europe, la production automobile a toujours été localisée dans quelques principaux pays fabricants que sont l'Allemagne, la France, le Royaume-Uni, la Tchécoslovaquie, la Suède et I'Italie. Or, avec I'ouverture de I'Union européenne à I'Est, des reconfigurations spatiales de l'industrie automobile sont en train de s'opérer. L'internationalisation des activités de production et de recherche et développement (R\&D), à I'œuvre dans le cadre d'une Europe élargie, nécessite de s'interroger sur les logiques générales qui guident cette recomposition de l'industrie automobile vers les PECO. Les principes décisionnels et les choix des constructeurs automobiles qui modifient la carte productive européenne constituent-ils une rupture ou la poursuite d'un processus inhérent au secteur?

\section{Les héritages de l'industrie automobile en Europe centrale et orientale}

M. Foucher (1993) a recensé les importantes bases productives dont disposaient ces pays à la veille de la chute du Mur. L'industrie automobile s'y était d'abord développée à partir d'une base industrielle autonome (ex : Skoda dans la région de Prague, Tatra en Moravie), firmes dont les créations sont contemporaines, à la fin du XIX et au début du $X X{ }^{e}$ siècle, de celles de I'Ouest. Le volontarisme industriel pendant la période communiste permit ensuite de développer d'autres marques nationales ou en coopération avec les pays frères. Au cours des années 1950 à 1970, ce furent, avec les Soviétiques, I'IMS roumain, frère du GAZ russe ; les camions roumains Steagul Rosu/Le Drapeau Rouge, la ARO communiste. Enfin d'autres marques " nationales " naquirent à partir d'accords de coopération sous licence avec les firmes occidentales comme FIAT en Yougoslavie (1955) ou en Pologne, ou avec des firmes allemandes (bus en Hongrie, camions RO-MAN en Roumanie), et plus tard, Renault en Roumanie (1969) avec la firme nationale Dacia et, en 1972 dans l'actuelle Slovénie, pour ne citer que les plus importants. Quelle que soit l'origine de ces bases industrielles, pendant la seconde moitié du $X X^{\mathrm{e}}$ siècle, les productions et les ventes ne furent cependant jamais comparables à celles des firmes de l'autre côté du Rideau de fer, ni sur le plan technologique, ni pour le renouvellement des modèles².

À la fin de la période socialiste, la production automobile dans les PECO ne dépassait pas $6 \%$ du marché mondial en 1989. À l'image de la fameuse Trabant de l'ex-RDA, la base productive était alors technologiquement dépassée et inappropriée pour produire des véhicules répondant aux standards exigés par la demande européenne. La transition vers un modèle capitaliste a engendré deux changements fondamentaux qui ont affecté l'industrie automobile dans les années 1990. Premièrement, la désintégration du $\mathrm{CAEM}^{3}$ a engendré une réorientation des échanges vers l'Europe occidentale. Deuxièmement, il s'est opéré une restructuration de l'appareil productif qui a eu pour conséquence, dans certains pays comme la Hongrie, la Pologne, la Slovénie et l'Estonie, la mise en place d'une politique intensive de privatisations qui ont été réalisées pour la plupart grâce à des capitaux extérieurs. Durant cette période, certaines firmes socialistes ont fait faillite, ont été réorganisées et restructurées, ou ont simplement été rachetées par des investisseurs étrangers ; ce fut le cas de Skoda (République tchèque) par Volkswagen. Ainsi, l'industrie automobile d'Europe centrale et orientale a expérimenté une transformation radicale à travers les investissements directs étrangers (IDE) et a été intégrée aux systèmes productifs de l'Europe occidentale et aux différents réseaux de distribution. Notons que ce processus d'internationalisation et d'intégration des périphéries a été similaire dans les autres marchés émergents de l'industrie automobile, comme en Espagne ou en Amérique latine à des périodes antérieures (Layan, 2000).

\section{Le nouveau départ de l'industrie automobile centre-européenne}

À la faveur des restructurations et des investissements étrangers, la production automobile totale en Europe centrale et orientale a augmenté, passant ainsi de 531000 véhicules produits en 1990 à 3489000 en 2007. D'après le Comité des constructeurs français de l'automobile (CCFA), la production de véhicules dans les PECO entre 2006 et 2007 a crû de $26,4 \%$, tandis que les immatriculations de voitures neuves sur la même période ont augmenté de 13,9\%. La production automobile dans les PECO a explosé et cette tendance tend à être aussi soutenue entre 2007 et 2010, alors que quatre grandes implantations de firmes ont eu lieu. Deux unités de production ont été ouvertes en Slovaquie au début 2006 (PSA Peugeot Citroën, Kia), une a été lancée en mars 2005 en République tchèque (Toyota/PSA Peugeot Citroën) et la dernière a été créée fin 2006 (Hyundai) toujours dans ce pays. On estime que ces quatre nouvelles implantations devraient augmenter la production annuelle des PECO d'environ 1,2 millions de véhicules. On remarque également qu'il existe une forte concentration des implantations des firmes automobiles dans une zone relativement restreinte comprenant la République tchèque, I'Ouest de la Slovaquie, l'Est de l'Allemagne et le Sud de la Pologne (fig. 5). Cette concentration des activités de production se 
traduit également par une croissance inégale de la production automobile (fig. 2) : la République tchèque, la Slovaquie et la Pologne sont en tête pour le nombre de voitures produites sur la période 1990-2006.

Si I'on compare la production automobile de I'Europe occidentale à celle de l'Europe centrale et orientale, on note une assez forte corrélation inverse. En effet, alors que, d'une manière générale, la production de véhicules baisse en Europe de l'Ouest, on assiste à une augmentation de la production dans les PECO. La figure 3 permet de mettre en regard cette évolution de la production automobile avec les phases du cycle de vie des produits. En effet, alors que globalement la production de voitures se stabilise en Europe de l'Ouest, on observe parallèlement une croissance importante des volumes de production de voiture dans les PECO (la production a plus que doublé !). Seule en Europe de I'Ouest, l'Allemagne a vu sa production augmenter dans le temps. Ce phénomène s'explique par le fait que les constructeurs automobiles allemands fabriquent des voitures haut de gamme qui ne peuvent être produites dans les pays d'Europe centrale et orientale. L'image de voiture de luxe, associée aux constructeurs automobiles allemands, explique pourquoi certaines marques ont choisi de maintenir leurs unités de production dans leur pays d'origine : par exemple, $90 \%$ des véhicules de la marque Audi sont fabriqués en Allemagne (Hertrich et Mayrhofer, 2007).

Les déterminants du déploiement de l'industrie automobile en Europe centrale et orientale : avantages compétitifs et processus d'intégration

Pour comprendre les phénomènes d'élargissement de l'espace productif, la première démarche consiste à observer dans quels États, différents de celui d'origine, les constructeurs automobiles fabriquent ou assemblent. Si l'on prend l'exemple allemand, Volkswagen fabrique des unités du modèle Passat en Slovaquie, tandis que Audi le fait avec le modèle TT en Hongrie. Concernant les groupes français Renault et PSA, une partie de leur production est déconcentrée également à I'étranger. Les délocalisations de tout ou partie des chaînes de production ont plusieurs explications : souvent, le constructeur automobile a racheté (voire parfois fait construire, mais c'est plus rare) une usine en Europe orientale pour y fabriquer une gamme de véhicules peu onéreux, avec la population locale comme débouché de ces modèles (tabl. 1). Si l'on compare le nombre d'immatriculations de voitures neuves, nous observons un accroissement du marché automobile national (une variation globale de $56,94 \%$ entre 2003 et 2006). On notera notamment la croissance très marquée des nouvelles immatriculations pour la Roumanie, la Bulgarie et la Lettonie, dont les $\mathrm{PIB} / \mathrm{hab}$. respectifs enregistrent de fortes croissances (fig.4 et 6).

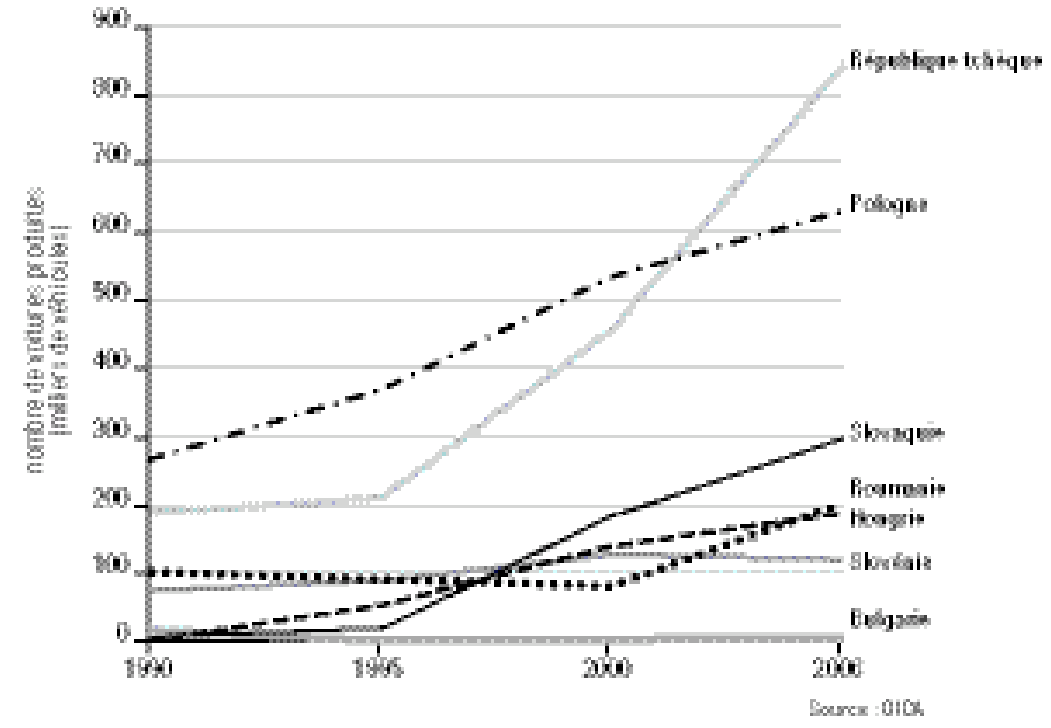

Figure 2 : Évolution de la production automobile en Europe centrale et orientale entre 1990 et 2006

Source : OICA

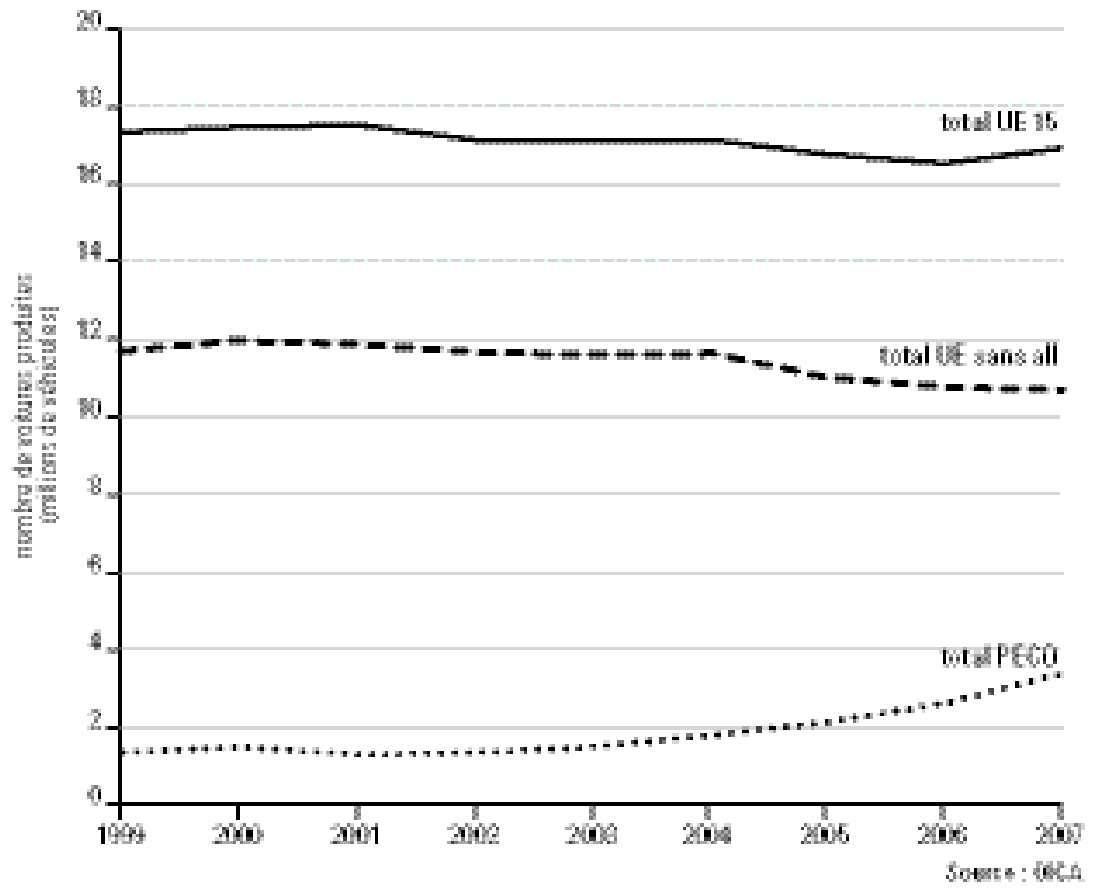

Figure 3 : Évolution de la production automobile en Europe entre 1990 et 2006 Source : OICA

Les véhicules produits (polyvalents et robustes) sont destinés au marché local ${ }^{4}$ avec des coûts de production très inférieurs ${ }^{5}$ à ceux observés en Europe de l'Ouest. II faut ajouter à cela que, d'une manière générale, les consommateurs ont globalement une image plutôt positive du constructeur s'installant dans le pays, car ce dernier est perçu comme un investisseur qui s'implique dans le développement du pays et qui favorise ainsi l'achat des voitures produites sur place. Aux coûts de production moins élevés dans les pays de l'Est viennent s'ajouter les avantages fiscaux que les États leur octroient, motivant ainsi les entreprises automobiles à s'installer sur leur territoire.
4 - Bien que le prix d'un véhicule ne diffère pas entre I'Europe de l'Ouest et l'Europe centre-orientale (exemple de la Logan - Dacia).

5 - Main-d'œuvre bon marché et flexible - technologies relativement basiques. 


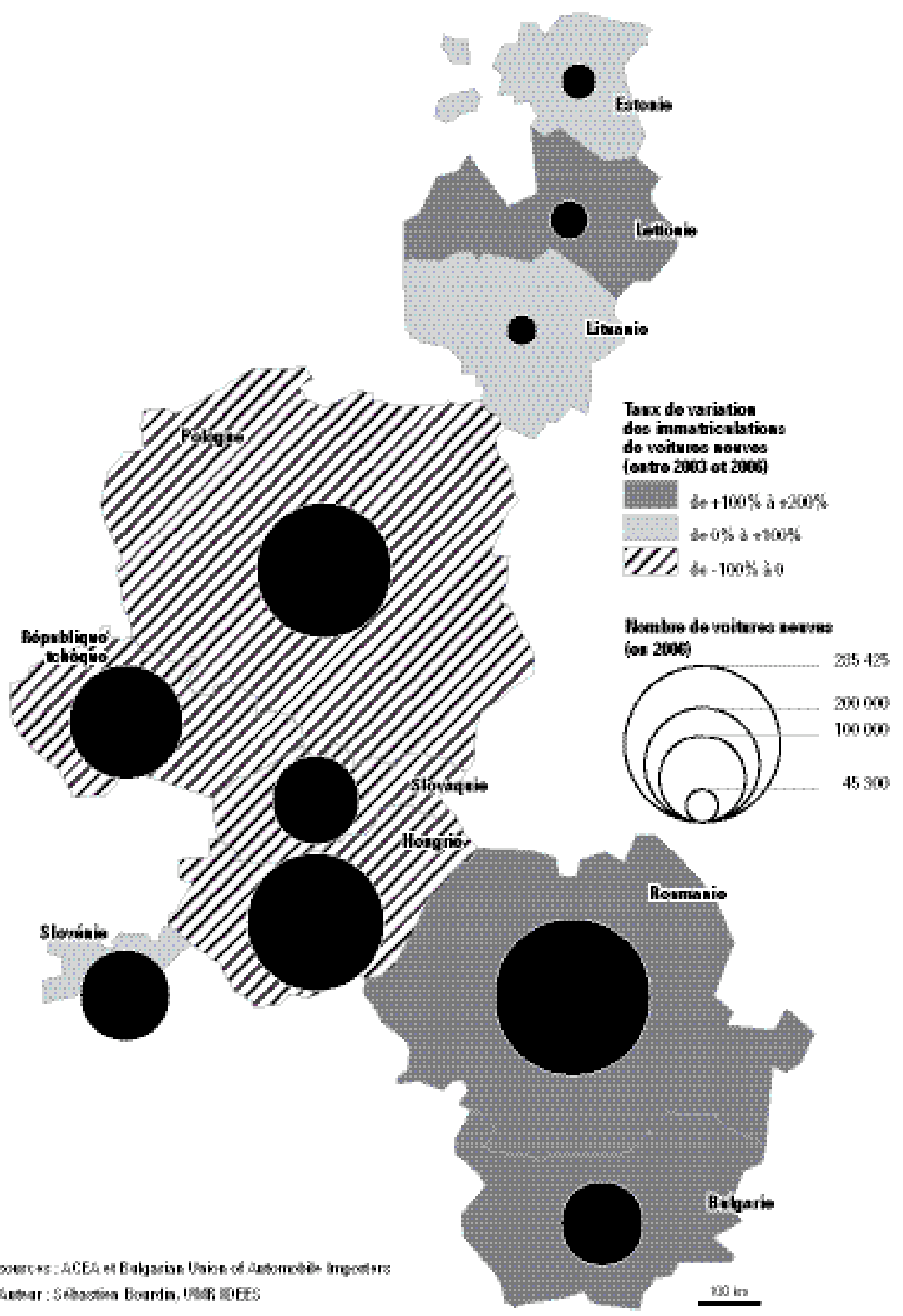

Figure 4 : Les immatriculations de voitures neuves dans les PECO

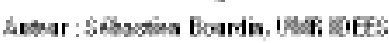

Conformément au cycle du produit, on assiste à la mise en place d'une nouvelle organisation spatiale des activités de production. Tandis que la fabrication des véhicules serait délocalisée dans les pays à faibles revenus, seules les activités à haute valeur ajoutée, comme la $R \& D$, le design ou encore la production de véhicules très haut de gamme, demandant une main-d'œuvre très qualifiée, seraient maintenues dans les pays d'Europe de l'Ouest.

L'approche théorique menée par J.H. Dunning (1988) nous permet d'apporter un éclaircissement sur la mobilité des productions qui s'opère actuellement dans l'industrie automobile. Deux points nous intéressent particulièrement dans le cadre de notre approche : d'une part, la Location approach explique les raisons des investissements directs effectués à l'étranger et, d'autre part, I'Industrial organization dont l'un des objectifs est de s'interroger sur les raisons qui poussent les firmes à s'installer à l'étranger. Les avantages des firmes à internationaliser leur production doivent alors être supérieurs aux coûts générés par cette dernière. Concernant la Location approach, J.H. Dunning apporte un certain nombre de réponses aux raisons des choix de localisation. Ainsi, il développe le modèle qu'il nomme ESP (Environment, Systems, Policies) pour comparer les différents pays et leur attractivité. II affirme que l'environnement économique, le système social et les politiques gouvernementales sont pris en compte dans les choix de localisation. Cette approche théorique privilégie l'étude des 
avantages d'un pays. Dans notre cas d'étude, tout en gardant la structure de la pensée de J.H. Dunning, nous souhaitons davantage appréhender les PECO comme un tout, partant du principe que ces pays disposent globalement d'avantages comparatifs similaires.

Les politiques de soutien à l'investissement, tout comme les politiques d'aménagement du territoire et de sauvetage des régions en déclin, relayées au sein de I'Union européenne par les transferts communautaires des fonds structurels, ont permis aux PECO d'offrir un cadre économique, social et institutionnel favorable à l'implantation de nouvelles firmes. Les instances de I'Union européenne n'ont pas un rôle neutre dans le développement industriel des périphéries de l'espace communautaire : en témoigne l'exemple de Volkswagen qui, en 1994, pouvait recevoir 230 millions d'écus, représentant près de $30 \%$ de I'investissement total (Layan, 2003), pour renouveler sa gamme de petits utilitaires produits à Val di Sangro (Abruzzes). Conformément au cadre théorique présenté par J.H. Dunning, force est de constater que les choix de localisation des principaux constructeurs automobile se sont tournés très rapidement vers les PECO. L'intégration dans I'Union européenne est assez rapidement apparue comme I'aboutissement du processus de transition. Elle a constitué un projet susceptible de mobiliser les énergies dans le sens d'une consolidation de la démocratie et de I'amélioration des résultats économiques et d'une plus grande cohérence territoriale. Ainsi, le degré d'ouverture des PECO s'est accru et les firmes multinationales de l'industrie automobile se sont fortement implantées à partir de la mise en place du processus d'intégration de ces pays. Le rapport établit par Win Kok sur l'élargissement de I'UE souligne très justement que "l'effet dynamique généré par le cercle vertueux de la croissance et des investissements favorise une croissance économique plus rapide au sein des nouveaux États membres après leur adhésion. Cependant, les expériences passées prouvent que cet élan dynamique n'a pas toujours été au rendez-vous. II sera largement tributaire des politiques adoptées par les nouveaux États-membres et par I'UE dans son ensemble " 6 .

Ceci conduit certains observateurs à replacer l'étude de ces mouvements de capitaux dans le contexte de l'intégration régionale du continent européen plutôt que dans celui du processus de mondialisation au sens large (Louis et Lepape, 2004). L'accession des populations de l'Est au statut de consommateurs de masse sur le modèle occidental traduit bien un système social recomposé, comme il est mentionné dans le modèle de Dunning. Le nombre d'immatriculations des deux pays ayant intégré I'UE en 2007 (Bulgarie et Roumanie) est un indicateur permettant de montrer les changements sociaux opérés (fig.4).

Aujourd'hui, les PECO sont parvenus à mettre sur pied une politique économique, sociale et territoriale favorable à l'accueil d'investissements

\begin{tabular}{|l|l|l|c|c|}
\hline Marque & Localisation & Pays & $\begin{array}{c}\text { Année } \\
\text { d'investissement }\end{array}$ & $\begin{array}{c}\text { Capacité de } \\
\text { production } \\
\text { en véhicules }\end{array}$ \\
\hline Suzuki & Esztergom & Hongrie & 1990 & 200000 \\
Renault/Revoz & Novo Mesto & Slovenie & 1991 & 180000 \\
Fiat & Bielsko-Biala & Pologne & 1991 & 250000 \\
VW/Skoda & M. Boleslav & Rep tchèque & 1991 & 450000 \\
Audi & Gyor & Hongrie & 1992 & 40000 \\
Volkswagen & Poznan & Pologne & 1993 & 120000 \\
Volkswagen & Bratislava & Slovaquie & 1993 & 350000 \\
GM/Daewoo & Craiova & Roumanie & 1996 & 22000 \\
Daewoo/FSO & Warsaw & Pologne & 1996 & 150000 \\
GM/Opel & Gliwice & Pologne & 1998 & 120000 \\
Renault/Dacia & Pitesti & Roumanie & 1998 & 100000 \\
PSA/Toyota & Kolin & Rep tchèque & 2002 & 300000 \\
PSA Peugeot/Citroën & Trnava & Slovaquie & 2003 & 450000 \\
Hyundai/KIA & Zilina & Slovaquie & 2004 & 300000 \\
Hyundai & Nosovice & Rep tchèque & 2006 & 300000 \\
\hline
\end{tabular}

Tableau 1 : Créations ou rachats d'unités de production automobile en Europe centrale et orientale

Source: PricewaterhouseCoopers Automotive Institute

On distinguera d'un côté les investissements de modernisation par rachat définitif par les firmes occidentales d'entreprises de l'Est avec lesquelles elles possédaient des accords de licence (Renault, Fiat, Volkswagen) ; de l'autre, des créations ex nihilo de nouvelles unités de production (ex : PSA/Toyota à Kolin et PSA à Trnava), qui s'amplifient au tournant des années 2000.

et permettant de développer leurs avantages compétitifs qui font qu'une firme automobile aura davantage tendance à ouvrir un nouvel établissement en Europe centre-orientale plutôt qu'en Europe de l'Ouest. Les PECO ont atteint un certain degré de maturité économique et politique et ont su mettre en place des structures favorisant le marché.

\section{Les cycles d'extension des aires productives}

À la lumière des faits exposés, il est possible d'affirmer qu'il s'opère une reconfiguration de la géographie de l'industrie automobile en Europe. Cette reconfiguration correspondrait à la troisième phase du déploiement territorial de l'industrie automobile sur le continent européen. On assiste bien à un processus d'intégration de ces périphéries dans le système productif européen. La phase initiale de déconcentration à l'intérieur des frontières nationales de chaque constructeur fut suivie par l'arrivée des constructeurs étrangers dans les périphéries ibériques et britanniques, à laquelle succède aujourd'hui la phase centreeuropéenne.

Sur la figure 5, trois ensembles territoriaux peuvent être identifiés. En premier lieu, émerge le cœur économique de l'Europe avec une forte présence des constructeurs automobiles français, allemands et italiens. Ce cœur industriel de l'Europe continue de mettre en avant ses atouts fondés sur des compétences en termes de qualification de la main-d'œuvre, de réseaux d'approvisionnement, de proximité des marchés et des centres de conception et de recherche. Le deuxième bassin concerne le pôle ibérique, qui s'étend du Sud de la France au Nord de l'Espagne et du Portugal, avec une présence de constructeurs automobiles français, allemands et américains. Ce pôle s'est développé à partir des années 1980, principalement en s'orientant dans la production de véhicules d'entrée de gamme (Fernandez et al., 2001) : ainsi, l'Espagne, pays peu industrialisé jusque dans les années 1970, est actuellement le troisième pays automobile
6 - Rapport de Win Kok à la

Commission européenne, Élargissement de l'Union européenne - résultats et défis, mars 2003 


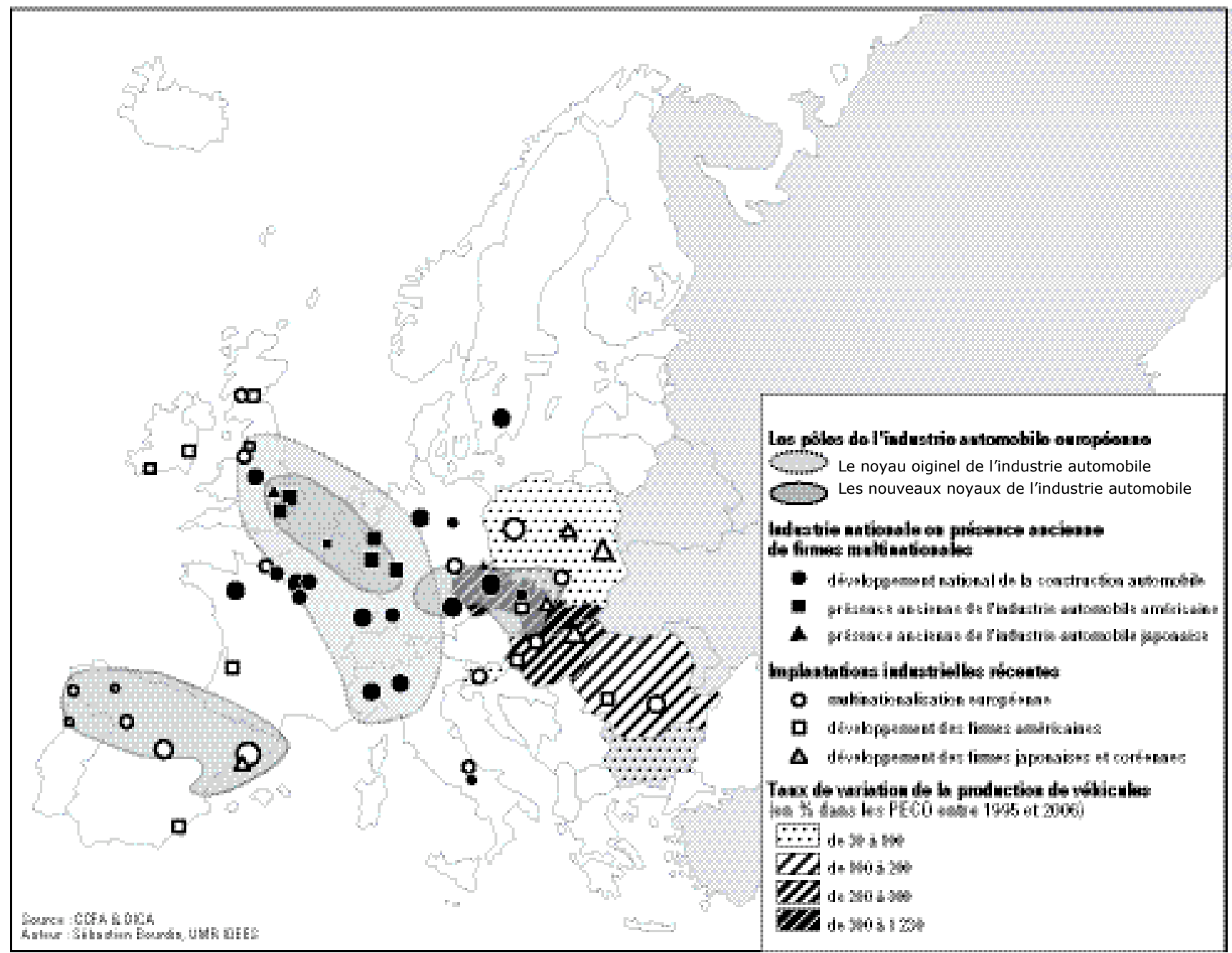

Figure 5 : Les cycles d'extension de la production automobile en Europe

7 - Fiat, GM, Opel et Daewoo en Pologne, Suzuki en Hongrie ; Volkswagen (VW) en République Tchèque ; Ford et Renault en Roumanie.

8 - Source : cabinet EulerHermes et article du Monde du 1/7/2006. européen et le cinquième mondial, avec plus de 3 millions de véhicules assemblés chaque année. Cet ensemble enregistre depuis peu une perte de compétitivité au profit du troisième pôle. $\mathrm{Ce}$ dernier a émergé en Europe centrale et orientale qui connait une forte croissance depuis les années 1990 résultant de l'arrivée massive d'IDE dans la filière automobile. II s'étend de I'Allemagne au Nord-Ouest de la Roumanie et est dominé par le constructeur Volkswagen. La concurrence entre les espaces de production tend à montrer en basculement du Sud vers l'Est de l'Europe (Lung, 2002). La restructuration radicale des PECO et leur intégration dans I'Union européenne leur a permis d'entrer dans le réseau européen de la production automobile. En quelques années, ces pays - et en particulier la Slovaquie et la République tchèque se sont affirmés comme le troisième pôle productif automobile en Europe. Les taux de croissance de I'industrie automobile y sont parfois spectaculaires avec, pour la République tchèque, une production multipliée par 3 et, pour la Slovaquie, une production multipliée par 12 .

Les constructeurs automobiles d'Europe de l'Ouest continuent d'adopter différentes stratégies dans ce troisième pôle. En effet, les PECO ont dû se spécialiser, d'une part dans la production massive de petites voitures ou de voitures d'entrée de gamme destinées au marché local (représentant par exemple $50 \%$ des ventes en Slovénie, en République tchèque et en Estonie) et à I'exportation?. D'autre part, certaines régions
d'Europe centrale et orientale se sont davantage concentrées vers un volume de production moindre mais de haute qualité qui trouve ses débouchés principalement dans les pays d'Europe occidentale (Layan, 2003 ; Pavlinek, 2001). S'il n'y a pas, à proprement parler, de délocalisations, on peut néanmoins parler de déplacement du centre de gravité de la production automobile européenne. En 2010, plus de 15\% des voitures du groupe PSA seront produites à l'Est, contre zéro en 2005. Les PECO ont, dans leur ensemble, exporté 217500 véhicules en 2005 , alors que la région était importatrice nette de plus de 400000 unités en 2000. Depuis 2007, la Slovaquie détient le record du monde de voitures produites par habitant (une pour six habitant $)^{8}$ Par rapport au modèle initial du cycle du produit, les dynamiques spatiales de I'industrie automobile se démarquent par l'absence de ruptures technologiques qui viendraient scander chaque nouvelle phase d'expansion au Sud ou à l'Est de I'Union européenne. On assisterait plutôt à la poursuite d'un processus d'élargissement des zones productives et de déplacement des volumes de production. Dans ce schéma, la translation actuelle vers les PECO ne constituerait qu'une nouvelle phase, s'inscrivant dans la continuité de processus inaugurés il y a deux décennies dans les périphéries méditerranéennes ou les régions industrielles en reconversion de I'Union européenne. 


\section{CONTINUITÉ DES SITES DE PRODUCTION ET CHANGEMENT DE SYSTÈME PRODUCTIF EN ROUMANIE}

La Roumanie a été longtemps considérée comme un pays spécialisé dans l'industrie lourde, notamment la sidérurgie et l'équipement ${ }^{9}$. L'intégration dans I'Union européenne, les possibilités d'immigration et l'arrivée d'entreprises étrangères, poussent aujourd'hui à la hausse des salaires. Les industries roumaines sont concurrencées par l'arrivée d'industries à plus forte valeur ajoutée, comme les industries automobiles (Ford et Renault) ou les nouvelles technologies (Nokia). Les bassins de main$d^{\prime} œ u v r e d^{\prime} u n$ secteur industriel sont alors progressivement remplacés par d'autres. Les constructeurs automobiles et les équipementiers qui avaient, dans un premier temps, investi dans les PECO les plus proches de l'Europe de l'Ouest ont, en marge de cette logique, également choisi la Roumanie. Si, comparativement, peu de voitures sont fabriquées en Roumanie, le secteur de I'industrie automobile est le plus dynamique du pays, en termes de production et d'emplois, grâce aux investissements des firmes multinationales Ford et Renault et à la production de pièces détachées destinées à être exportées dans les différentes usines d'assemblage de la Logan dans le monde. Ces implantations, qui se surajoutent à un tissu industriel hérité du schéma socialiste, posent la question du mode d'organisation de I'espace des firmes au niveau local et du modèle productif de référence (fordisme ? toyotisme ?) qui y a été transposé. Les lignes qui suivent ont pour objet de montrer comment ces investissements s'ajustent dans les territoires roumains avec I'héritage du substrat industriel et social des combinats de l'époque socialiste.

\section{La place de la Roumanie dans la stratégie d'investissement des constructeurs}

En choisissant la Roumanie, les constructeurs automobiles ont investi dans un pays où la maind'œuvre est bon marché (avantage comparatif) et bien formée (avantage compétitif). En rachetant des entreprises déjà spécialisées dans la production automobile, Ford et Renault espèrent bénéficier d'une plate-forme productive, d'une main-d'œuvre qualifiée et d'un coût du travail très peu onéreux (salaire mensuel moyen net de 180 euros en 2007 selon I'Institut national des statistiques roumain). Bien entendu, les coûts salariaux ne constituent pas le facteur unique, sinon les firmes iraient dans les pays à très faibles coûts salariaux (les pays asiatiques émergents par exemple, les moins chers du monde). De plus, on peut estimer que les différences salariales entre l'Est et l'Ouest de l'Union européenne vont se réduire, conformément au processus de convergence selon lequel il y aurait un " rattrapage progressif " du niveau de vie moyen de l'Est vers celui de l'Ouest.
Enfin, la Roumanie est une zone à fort potentiel de croissance, puisque son produit intérieur brut a toujours augmenté de plus de $4 \%$ depuis 2001 et, depuis 2004, le taux d'inflation n'a pas dépassé la barre des $10 \%$. Alors, même si la Roumanie connaît encore une forte pauvreté avec un $\mathrm{PIB} /$ habitant de 3740 euros $^{10}$ (inférieur à la moitié de la moyenne de l'Union européenne à 27) et des salaires qui restent inférieurs à 180 euros par mois, les Roumains vivent ce qu'on appelle une " consommation de rattrapage " (Lhomel, 2004). On peut le constater dans la figure 6 : en 2006, les Roumains ont acheté 3,5 fois plus de voitures qu'ils n'en achetaient en 2000. De plus, avec 21,5 millions d'habitants en 2008 , la Roumanie est le deuxième marché de consommation potentiel derrière la Pologne pour les PECO, même si le pays connaît un déclin démographique majeur avec un million d'habitants en moins depuis sept ans ${ }^{11}$.

La figure 6 représente l'évolution de la production des automobiles construites en Roumanie et l'évolution de la totalité des ventes de voitures neuves dans le pays. II y avait trois constructeurs qui produisaient en Roumanie : Aro, Daewoo et Dacia. Aro était une entreprise roumaine de production de voitures tout-terrain, elle a fermé en 2005, les ventes étant inférieures à une centaine de voitures. Daewoo, anciennement Olcit-Citroën, a produit en Roumanie jusqu'en 2008, mais elle a récemment été rachetée par Ford qui a prévu de produire dans cette usine des voitures de petit modèle. Quant à Dacia, c'est la voiture de marque roumaine qui existe depuis 1968.

L'environnement économique roumain est donc davantage conforme aux normes du marché international depuis le début des années 2000, mais le fait que la Roumanie n'ait pas pu intégrer I'Union européenne dans la première vague d'adhésion des PECO a probablement fait craindre aux potentiels investisseurs des risques d'instabilité politique, sociale et économique, des " risques-pays " peu propices à l'environnement financier et commercial. II a fallu attendre 2004 et la promesse d'adhésion à l'Union européenne pour que les investissements étrangers augmentent véritablement et dépassent la barre des 5 milliards d'euros. Cela explique probablement en partie pourquoi les firmes ont choisi d'investir plus tardivement en Roumanie que dans d'autres PECO.

C'est également à partir de 2004 que les politiques économiques ont favorisé les investissements étrangers, avec l'instauration d'un taux unique de $16 \%$ pour l'impôt sur les entreprises ${ }^{12}$. Les firmes peuvent également bénéficier de nombreuses facilités comme l'exemption du paiement des taxes douanières pour les biens neufs importés et nécessaires à la réalisation de l'investissement ou à une déduction fiscale. En 2007, la Banque mondiale a classé la Roumanie 49 e sur les 175 pays étudiés dans un rapport sur l'environnement
9 - Rôle qui lui avait été donné dans le cadre de la CAEM (Conseil d'aide économique mutuelle).

10 - Source : INS

11 - II y avait 22500000 habitants en 2001.

12 - $25 \%$ auparavant. 


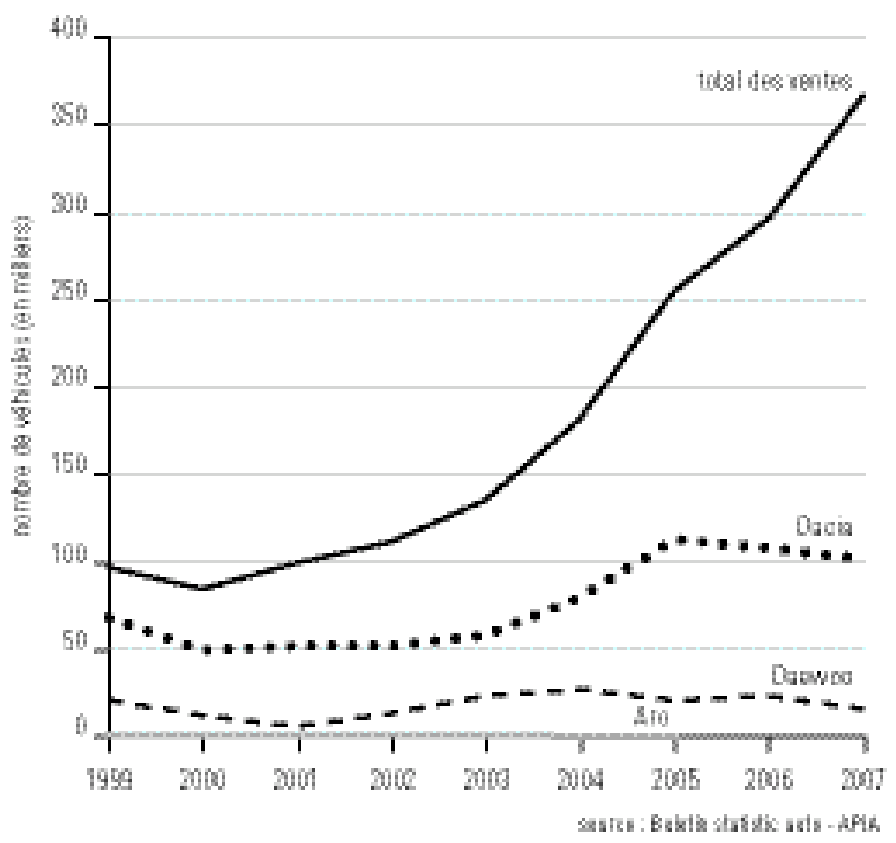

Figure 6 : Évolution des ventes de voitures neuves depuis 1999 et vente des productions des marques nationales

13 - Rapport de la Banque Mondiale "Doing Business 2007".

14 - Le judet roumain est l'équivalent du département français.

15 - La période des grandes privatisations a duré de 1996 à 2000 lorsque $E$.

Constantinescu était Président de la Roumanie.

16 - Trois combinats ont été créés à Pitesti entre 1966 et 1968 : une usine de moteurs électriques, un combinat pétrochimique et l'usine automobile de Pitesti (devenue aujourd'hui Dacia).

17 - Pour plus d'informations sur les villes roumaines, cf. Emsellem, 2001.

18 - C'est seulement en 1996 que la commune de Colibasi s'est appelée Mioveni et a obtenu le statut de ville. des affaires ${ }^{13}$. La Banque mondiale notait une progression de la Roumanie dans cinq domaines : I'obtention d'un permis de construire, I'obtention d'un prêt, la protection des investisseurs, la facilité des échanges commerciaux et le dépôt de bilan. Néanmoins, en ce qui concerne le recrutement du personnel, l'enregistrement d'une entreprise, le paiement des impôts et la procédure de faillite, le classement de la Roumanie restait passable à l'échelle mondiale.

Ainsi, la Roumanie possède certains atouts pour attirer les firmes étrangères : I'environnement économique est favorable, les mutations sociales sont attractives pour les firmes étrangères depuis l'entrée du pays dans I'Union européenne et les politiques gouvernementales favorisent l'investissement en minimisant les risques financiers.

Les investissements sont donc le résultat d'un arbitrage entre les coûts générés par l'installation à l'étranger et les avantages obtenus sur les coûts de production. Ainsi, les constructeurs Ford et Renault ont choisi de se localiser en Roumanie en optant pour des investissements de type " acquisition ". Ford a racheté I'usine Daewoo située à Craiova (Sud de la Roumanie) et Renault I'usine Dacia dans le plateau du Sud des Carpates méridionales, à une centaine de kilomètres de la capitale roumaine. C'est ce second exemple qui sera développé.

\section{Comment l'industrie construit l'espace dans le judet $^{\mathbf{1 4}}$ de l'Arges ?}

Renault a racheté Dacia en 1998, lors de la période des grandes privatisations ${ }^{15}$. Cette fusionacquisition a été présentée comme le prolongement de relations antérieures puisque les premières voitures de la marque Dacia ont été créées sous licence Renault à partir de 1968. Avec 28000 employés, I'usine Dacia était I'un des principaux employeurs ${ }^{16}$ de la région autour de Pitesti (fig. 7) à l'époque communiste. Lorsque l'usine a été créée, de nombreux salariés sont arrivés sur le site pour y travailler, entraînant un effet moteur sur toute la région. À l'échelle de la Roumanie, l'évolution de la ville de Pitesti a été la plus impressionnante en ce qui concerne l'évolution de la population, puisqu'en 1948 c'était une petite ville de 29000 habitants et qu'en 1992, il y avait plus de 184000 habitants ${ }^{17}$.

Le fait que Renault ait racheté Dacia lui a permis de consolider une place de leader sur le marché local et de se rapprocher des marchés voisins et, en achetant Dacia, Renault rachète la marque nationale, celle que les Roumains préfèrent acheter, avec une image de robustesse et de solidité. Le groupe Renault possède déjà Nissan et, en ayant cette troisième marque, il est moins sensible aux fluctuations du marché mondial, puisque le volume des ventes et la diversification géographique lui permettent, en théorie, d'amortir les difficultés.

Cependant, ce n'est pas le pays, dans sa globalité, qui est attractif pour les entreprises automobiles, mais certains sites bien déterminés. On assiste à une polarisation des localisations des industries automobiles et des sous-traitants, à la création d'un " noyau industriel " (Martin et Sunley, in Benko et Lipietz, 2000) situé sur un territoire étroit autour de I'usine Dacia. Sur la figure 7 sont représentés les établissements et les entreprises qui se sont installés après 1998, après la fusion entre Dacia et Renault. Selon les données dont nous disposons (Association des constructeurs automobiles de Roumanie, ACAROM), 70 établissements se sont installés en Roumanie depuis cette acquisition, soit deux fois plus de sous-traitants qu'avant le rachat de Dacia par Renault, et 9000 emplois ont été créés chez les sous-traitants automobiles depuis 1998, dont 4000 dans I'Arges (judet où est situé Dacia), 1300 à Bucarest et à Timifloara et un peu moins de 1000 à Sighifloara. On peut constater, en observant la carte, une agglomération d'une vingtaine de soustraitants dans le judet de l'Arges et environ vingtcinq dans le judet de I'Isov (département autour de Bucarest).

Une logique d'agglomération apparaît dans les choix de localisation des sous-traitants et des équipementiers, la main-d'œuvre locale étant déjà formée aux métiers de l'automobile. L'histoire de I'industrie automobile dans le village de Colibasi date de 1968. Lorsque les dirigeants roumains ont choisi de diversifier l'industrie nationale en se retirant du CAEM, ils ont décidé que la première usine de production automobile du pays se situerait à Colibasi (actuellement Mioveni) ${ }^{18}$, là où il y avait une usine de pièces mécaniques pour camions destinées aux usines de Brasov. À partir de 1968 , le village de Colibasi a évolué au rythme de l'usine, la population du village et des villages voisins a augmenté selon les besoins de maind'œuvre de I'usine : il y a, à Mioveni, ce que A. Marshall appelle une "professionnalité ambiante ", entretenue par les formations scolaires locales qui sont, en partie, spécialisées dans les métiers de l'automobile. II règne donc à Mioveni une " atmosphère industrielle " favorable à l'implantation des entreprises sous-traitantes de 

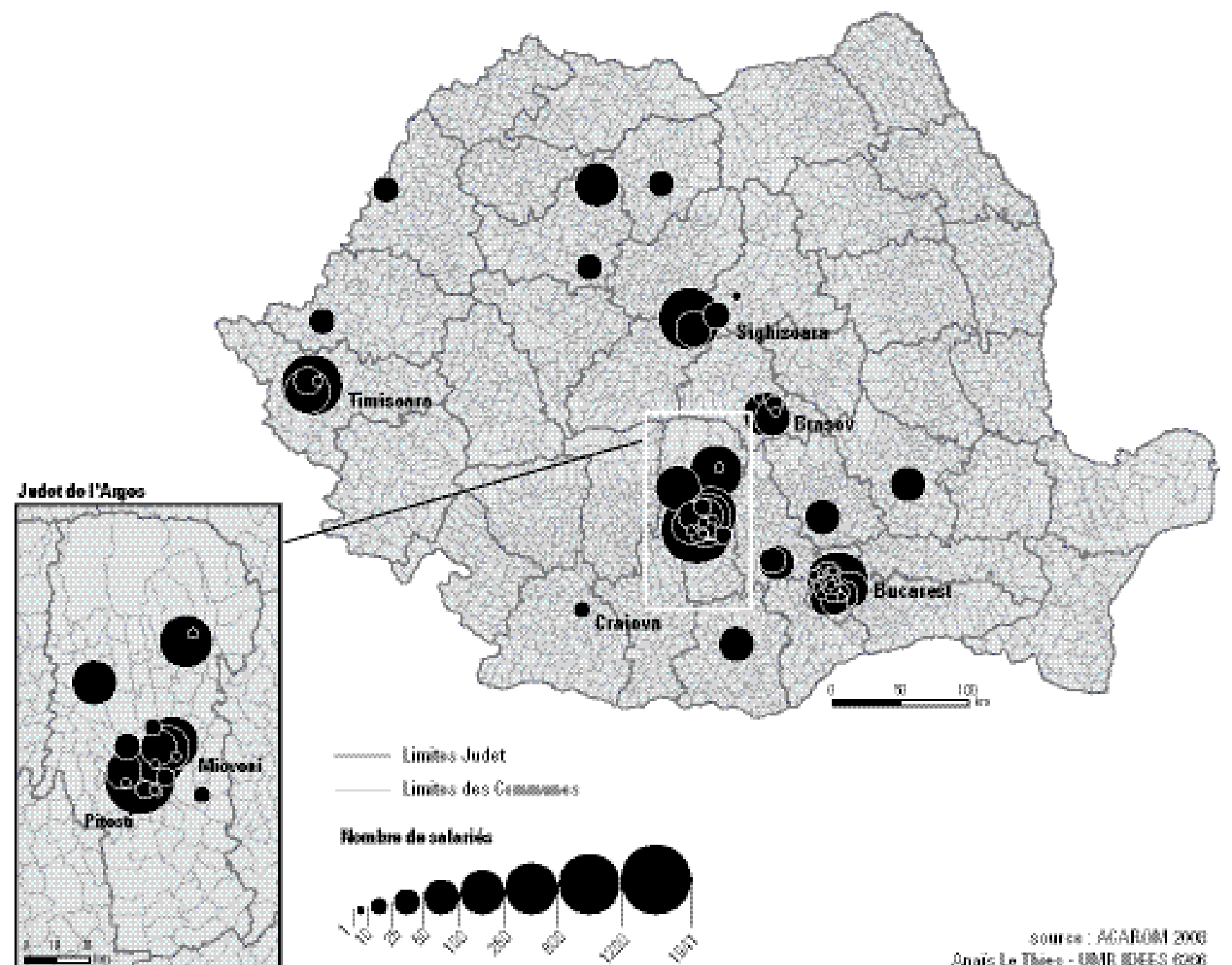

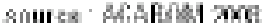
Anais Lo Thet - UAR WHES titr

Figure 7 : La sous-traitance automobile en Roumanie

Dacia-Renault. Dans le cas de I'industrie automobile, on ne parle pas de district industriel, puisque les entreprises sous-traitantes sont dépendantes d'un seul donneur d'ordre, mais de complexe industriel de type pyramidal (Scott, 2001) puisque le constructeur automobile se tient au sommet en étant le donneur d'ordre et le seul à la fin de la chaîne de production. Cependant si un " noyau industriel " se forme dans l'Arges, $d^{\prime}$ autres territoires roumains sont attractifs pour les sous-traitants, notamment autour de Bucarest, Brasov et Timisoara.

\section{Le changement du système productif dans le judet de l'Argefl}

Afin d'expliquer l'agglomération des sous-traitants et des équipementiers, il est nécessaire d'expliquer le changement de système productif lorsque Renault a racheté Dacia en 1998 . Si la soustraitance avait débuté à partir de 1990, I'usine Dacia fabriquait encore une grande partie de l'automobile, y compris les différentes pièces détachées, et seules $20 \%$ des pièces étaient importées avant 1999. Renault a transformé ce mode de production en passant " du combinat socialiste à l'entreprise capitaliste " (von Hirschhausen, 1996). L'établissement comptait encore 28000 employés en 1998 et, suite à
I'externalisation des activités de production, il n'y en avait plus que 12000 en 2004. Le constructeur français a transféré les opérations d'assemblage en amont, réalisées auparavant par l'usine Dacia, vers les fournisseurs. Comme nous le voyons dans la figure 7, des équipementiers se sont rassemblés autour de Mioveni, dont six sur une " zone industrielle fournisseur " (ZIF), sur la plateforme industrielle de Mioveni. La proximité entre I'usine d'assemblage et les équipementiers a permis de pratiquer un mode de production à flux tendus, rendu possible grâce à la proximité et à la flexibilité du travail. Ce mode d'organisation, le toyotisme, est aujourd'hui le plus répandu dans I'industrie automobile mondiale, il permet au constructeur d'adapter son niveau de consommation intermédiaire à celui de la production, il n'a donc plus à supporter le coût du stockage. M.G. Cedillo-Campos (2004) présente le toyotisme comme un facteur primordial de réussite de l'internationalisation avec la mise en place d'un système qui aboutit à un avantage compétitif collectif grâce à la synchronisation entre les sous-traitants et le rythme de production du constructeur automobile. Cet objectif a été rapidement mis en place sur la plate-forme de Mioveni puisque le constructeur français, moteur de l'activité économique locale, a pu obtenir de ses fournisseurs l'exécution de commandes à très 
court terme. Ce mode d'organisation peut cependant montrer rapidement ses limites, notamment en cas de grève, comme lorsqu'en mars 2008, les salariés de Dacia ont fait 18 jours de grève pour réclamer une augmentation de salaire.

Ainsi, les industries sous-traitantes automobiles se sont rapprochées le plus possible de leur donneur d'ordre (cf. fig. 7) et Renault a construit autour de Mioveni une nébuleuse d'entreprises. En 2008, il y a 136 sous-traitants et équipementiers automobiles en Roumanie (ACAROM), 37 sont situés dans l'Arges, dont six dans la zone industriel fournisseurs. Selon les chiffres fournis par Dacia, 4000 salariés travaillent dans la ZIF de Mioveni, 30000 emplois dépendent de Dacia dans le judet de l'Arges et 120000 dans toute la Roumanie. L'automobile, avec la présence de Renault notamment, est donc un grand pourvoyeur d'emplois pour le pays. L'arrivée massive des sous-traitants a permis au judet de l'Arges de devenir une plaque tournante pour l'exportation des voitures et de composantes automobiles vers les pays de la CEl et d'Europe du Sud.

La Roumanie occupe une place spécifique dans la nouvelle organisation spatiale des activités liées à I'industrie automobile européenne. La marque nationale historique Dacia contribue largement à cette nouvelle donne. En effet, le groupe Renault en faisant l'acquisition du combinat, a voulu spécialiser le site industriel roumain dans une voiture à bas coût, destinée au marché local dans un premier temps, pour ensuite être exportée vers les différents continents où se trouvent les usines d'assemblage de la Logan (Maroc, Inde, Iran, Russie, Brésil, Afrique du Sud et Colombie). De même, la firme Ford a choisi de spécialiser son site de production roumain dans un petit modèle destiné au marché local. Les investissements des groupes automobiles Renault et Ford en Roumanie contribuent ainsi à l'élargissement des zones de production vers l'Europe de l'Est, mais en faisant de ce pays la plate-forme européenne des véhicules universels à bas coût.

\section{CONCLUSION}

Les recompositions qui ont suivi la chute du Mur de Berlin, loin d'induire des reconfigurations complètes des usages fonctionnels des lieux, aboutissent, par bien des aspects, à des situations d'hétérotopie. Qu'il s'agisse de créations ex nihilo ou de rénovation d'une plate-forme industrielle ancienne, le redéploiement récent de la filière automobile en Europe centrale et orientale illustre I'utilisation différentielle des territoires au sein de l'espace communautaire, et le lancement d'une nouvelle séquence dans le, déjà long, cycle de vie du produit automobile. Cette nouvelle phase s'accompagne, à l'intérieur de chaque espace, de l'émergence de systèmes territoriaux hybrides, au sein desquels on n'a pas fait complètement table rase du passé, mais où les process industriels bien rôdés des grands groupes automobiles se greffent sur des structures pré-existantes.
À défaut de l'achèvement complet de cycles indépendants par grands ensembles continentaux, comme le postule le modèle initial du cycle du produit, se manifestèrent des séquençages partiels, qui atteignirent leur maximum lorsque les capacités (production/consommation) des marchés nationaux furent atteintes. Les PECO sont conformes à ce schéma en y ajoutant une nuance. Le déploiement actuel se greffe sur un ancien cycle fordiste avorté du fait de l'économie de pénurie de I'époque communiste, représentative de la défaillance de l'ancien système. Au lieu de laisser émerger des concurrents dans de nouveaux pays adhérents, les principaux constructeurs ont bloqué cette évolution en faisant des PECO un nouvel ensemble dans leurs systèmes productifs. De plus, avec le cas de la Roumanie et de la Logan de Renault-Dacia, une hiérarchisation des territoires semble s'esquisser au sein des PECO, associant coûts salariaux, type de véhicule et niveau de consommation du pays. Sur les territoires centreeuropéens s'enchevêtrent ainsi trois temporalités : celle de la translation des productions des constructeurs de I'Ouest à la faveur des avantages compétitifs issus de l'effondrement de l'ancien système ; celle de la reconversion des bassins de main-d'œuvre hérités des anciens combinats ; et enfin, celle de la diffusion des normes communautaires de consommation. La stabilité apparente du nouvel environnement économique, la synergie des investissements directs étrangers, la volonté des firmes multinationales automobiles de transférer leur savoir-faire et leurs technologies et, enfin, les politiques territoriales et fiscales mises en place nous laissent penser que ce pôle centre-oriental est voué à s'affirmer encore davantage à l'avenir.

\section{BIBLIOGRAPHIE}

AYDALOT P., 1976, Dynamique spatiale et développement inégal, Paris, Ed Economica, 336 p.

BENKO G. et LIPIETZ A., 2000, La richesse des régions, Paris, PUF, $564 \mathrm{p}$.

CEDILLO-CAMPOS M.G., 2004, Système industriel et système territorial, thèse de doctorat, Université de Paris Val-de-Marne, $404 \mathrm{p}$.

CHANARON J.L. et LUNG Y., 1995, L'économie de I'automobile, Paris, La découverte, $216 \mathrm{p}$.

DUNNING J.H., 1988, International Business in a Changing World Environment, in Multinationals, Technology an Competitiveness, London, Unwin Hyman Ltd., $280 \mathrm{p}$.

DUNNING J.H., 2001, The Eclectic (OLI) Paradigm of International Production: Past, Present and Future, International Journal of the Economics of Business, Volume 8, Issue 2, July, p. 173-190.

ELISSALDE B., DOMINGO J., 1994, L'espace mondial depuis les années 30, Paris, Bréal , 256 p. 
EMSELLEM K., 2001, Les petites villes dans le système de peuplement de la Roumanie, thèse de doctorat, Université Panthéon-Sorbonne (Paris), $520 \mathrm{p}$.

FERNANDEZ ENRIQUE de M. et al., 2001 Localisation et délocalisation de la production dans le secteur automobile : possibles effets dans le cas espagnol, $9^{e}$ rencontre internationale $d u$ GERPISA, 7-9 Juin, Paris, France, p. 1-14.

FOUCHER M., 1993, Fragments d'Europe, Paris, Fayard, $328 \mathrm{p}$.

GURAN-NICA L., 2002, Investitii straine directe, Dezvoltarea sistemului de aflezari din România, Bucuresti, Editura Tehnica S.A., 176 p.

HERTRICH S., MAYRHOFER U., 2007, AUDI AG : un succès signé made in Germany, Regards sur l'économie allemande, № 80 , p. 23-28.

HIRSCHHAUSEN B. von, 1996, Du combinat socialiste à l'entreprise capitaliste, Paris L'Harmattan, $252 \mathrm{p}$.

LAYAN J.B., 2000, The integration of peripheral markets: a comparison of Spain and Mexico, in HUMPHREY J., LECLER Y. and SALERNO M. (eds), Global Strategies and Local Realities: The Auto Industry in Emerging Markets, London, Macmillan, p.43-63.

LAYAN J-B., 2003, L'intérêt stratégique des périphéries du système automobile européen, $9 e$ rencontre internationale du GERPISA, 11-13 Juin, Paris, France, p. 1-24.

LHOMEL E., 2004, Roumanie 2003-2004, Sur la dernière ligne droite?, Le Courrier des Pays de
I'Est, n¹044, juillet-août, La Documentation Française, p. 185-201.

LOUIS O., LEPAPE Y., 2004, Les IDE, un facteur crucial de l'insertion des pays d'Europe centrale et orientale dans l'économie européenne, Annales des Mines, nov., p. 35-40.

LUNG Y., 2002, La nouvelle géographie du système automobile européen, $10^{e}$ rencontre internationale du GERPISA, 6-8 juin, Paris, France, p. 20-36.

MUNDELL R., 1971, Monetary Theory: Interest, Inflation and Growth in the World Economy, Pacific Palisades, Goodyear, Californie,190 p.

PAVLINEK P., 2001, The Central and East European Automotive Industry Restructuring, Slavic Research Center, n9 ${ }^{\circ}$ décembre, p 34-67.

REY V. et alii, 2007, Atlas de la Roumanie, Paris, La Documentation Française, $180 \mathrm{p}$.

SCOTT A. J. , 2001 Les régions et l'économie mondiale, Paris, L'Harmattan, $188 \mathrm{p}$.

VELTZ.P., 1996, Mondialisation, villes et territoires, Paris, PUF, $262 \mathrm{p}$.

VERNON R., 1966, International investment and international trade in the product-cycle, Quartely journal of Economy, may, p. 190-207.
Adresse des auteurs

UMR CNRS IDEES

Faculté LSH de Rouen

7 rue Thomas Becket 76821 Mont Saint Aignan cedex.

sebastien.bourdin@univrouen.fr

anais.lethiec@univ-rouen.fr bernard.elissalde@univrouen.fr 